\title{
Survival after alcohol septal ablation versus conservative therapy in obstructive hypertrophic cardiomyopathy
}

\author{
Yin-Jian Yang ${ }^{1}$, Chao-Mei Fan ${ }^{1}$, Jin-Qing Yuan ${ }^{2}$, Shu-Bin Qiao ${ }^{2}$, \\ Feng-Huan $\mathrm{Hu}^{2}$, Xi-Ying $\mathrm{Guo}^{1}$, Yi-Shi $\mathrm{Li}^{1}$
}

${ }^{1}$ Key Laboratory of Clinical Trial Research in Cardiovascular Drugs, Ministry of Health, Fuwai Hospital, National Center for Cardiovascular Diseases, Chinese Academy of Medical Sciences and Peking Union Medical College, Beijing, China

${ }^{2}$ Department of Cardiology, Fuwai Hospital, National Center for Cardiovascular Diseases, Chinese Academy of Medical Sciences and Peking Union Medical College, Beijing, China

\section{Abstract}

Background: The impact of alcohol septal ablation (ASA) on the survival of patients with drug-refractory obstructive hypertrophic cardiomyopathy (HCM) remains unresolved. The aim of this study was to compare survival after ASA vs. conservative therapy.

Methods: We studied a consecutive cohort of 274 patients with severe drug-refractory obstructive HCM, 229 in ASA group and 45 in conservative group. The primary endpoint was a composite of all-cause mortality and aborted cardiac arrest.

Results: With a median follow-up of 4.3 years, primary endpoint occurred in 13 (5.7\%) patients in the ASA group, and 8 (17.8\%) patients in the conservative group. The 5- and 10-year survival free from primary endpoint of the ASA group (94.5\% and 93.0\%, respectively) was significantly better than that of the conservative group $(78.3 \%$ and $72.2 \%$, respectively, log-rank $p=0.009$ ). Independent determinants of primary endpoint were ASA therapy (hazard ratio [HR] 0.22; 95\% confidence interval [CI] 0.08-0.60; $p=0.003$ ) and maximal septal thickness (HR 1.14; 95\% CI 1.03-1.27; $p=0.011$ ).

Conclusions: In patients with severe drug-refractory obstructive HCM, survival after ASA is favorable and better than that of conservative therapy. ASA seems to improve survival. (Cardiol J 2015; 22, 6: 657-664)

Key words: obstructive hypertrophic cardiomyopathy, alcohol septal ablation, medical therapy, conservative therapy, survival

\section{Introduction}

Hypertrophic cardiomyopathy $(\mathrm{HCM})$ is the most common genetic heart disease of Mendelian Inheritance, with a prevalence of $0.2 \%$ in the general population [1]. Left ventricular outflow tract obstruction (LVOTO), defined as a resting or provocative intracavity pressure gradient of $\geq 30 \mathrm{~mm} \mathrm{Hg}$, presents in about $70 \%$ of patients with $\mathrm{HCM}$ [2]. This hemodynamic disorder is associated with a variety of limiting symptoms and worse prognosis [3]. Medications (i.e. beta-blockers,

Address for correspondence: Chao-Mei Fan, MD, 167 Beilishi Rd, Xi Cheng District, Beijing, China, 100037, tel: +86-010-8839-6298, fax: +86-010-6835-4535, e-mail: fancm2004@126.com

Received: 01.04.2015

Accepted: 11.05.2015 
non-dihydropyridine calcium channel blockers, and disopyramide) are recommended firstly for symptomatic patients, but many patients cannot benefit from medical therapy. For severe drug-refractory symptoms that are attributable to LVOTO, invasive therapies such as alcohol septal ablation (ASA) and myectomy can be used to relieve LVOTO and improve symptoms $[4,5]$.

Studies have demonstrated that survival rates after ASA and myectomy in experienced centers were similar [6-8]. And, as survival after myectomy, survival after ASA is also comparable with that of general population $[8,9]$. These data support the notion that ASA may be another intervention which improves survival of patients with obstructive HCM and severe drug-refractory symptoms. Direct comparisons of survival after ASA and medical therapy will extend evidence to support this notion. There are studies showing better survival rates after ASA vs. medical therapy as well [10-12]. However, as there were inherent differences between the conservatively treated patients and patients undergoing invasive therapies with regard to pressure gradient and symptoms in these studies [10-12], the impact of ASA on the survival of patients with HCM, severe drug-refractory symptoms and significant LVOTO remains unsolved. This study will compare the survival of patients undergoing ASA vs. a group of conservatively treated patients with comparable pressure gradient and limiting symptoms.

\section{Methods}

\section{Study population}

This study consecutively enrolled adult ( $\geq 18$ years of age at initial presentation to Fuwai Hospital) patients with HCM, severe limiting symptoms (New York Heart Association [NYHA] functional class III/IV, Canadian Cardiovascular Society [CCS] class III/IV, or repetitive and disabling effort-related syncope) despite or unable to tolerate optimal medication treatment (beta-blockers [titrated to a resting heart rate of approximately $60 \mathrm{bpm}$ ], verapamil [maximum $480 \mathrm{mg} /$ day], diltiazem [maximum $360 \mathrm{mg} /$ day]), a pressure gradient of $\geq 50 \mathrm{~mm} \mathrm{Hg}$ at rest or with provocation (or both), and without a history of previous ASA, myectomy or dual chamber (DDD) pacing before their first admission to Fuwai Hospital between January 2002 and December 2012. The diagnosis of HCM was based on the presence of left ventricular maximum wall thickness $\geq 15 \mathrm{~mm}$ in the absence of another disease capable of producing the degree of hyper- trophy [13]. Echocardiographic data were obtained as previously described [14]. Left ventricular outflow tract (LVOT) pressure gradient was assessed using continuous wave Doppler echocardiography and cardiac catheterization [14-16].

Patients were classified into ASA and conservative groups based on the treatment strategies they received at their first admission to our hospital. The ASA group included patients who underwent ASA at their first admission. The conservative group included patients who were 1) indicated to undergo ASA but refused any invasive therapies to treat LVOTO; or 2) consented to receive ASA, but unsuitable for it because of no appropriate septal branches applicable, and refused myectomy and DDD pacing. Patients refused one or more invasive therapies for their fear of operation risk or economic reasons. For patients in the conservative group, only medications were used to treat LVOTO-related limiting symptoms.

If a patient was treated conservatively because of any of the following conditions, which would the patient unsuitable for ASA and essentially not comparable to patients in the ASA group, then they were excluded: severe coronary artery disease, acute myocardial infarction (MI), mid-ventricular obstruction, extreme hypertrophy $(\geq 30 \mathrm{~mm})$, diffused hypertrophy, moderate-to-severe mitral regurgitation, renal dysfunction, hyperthyroidism, hypothyroidism, liver dysfunction, malignant tumor, blood disease, massive pericardial effusion, Alzheimer's disease, recent stroke, severe hypertension, severe chronic obstructive pulmonary disease, or advanced age.

\section{Alcohol septal ablation}

All patients who received ASA presented a LVOT pressure gradient of $\geq 50 \mathrm{~mm} \mathrm{Hg}$ at rest or with provocation, associated with drug-refractory NYHA functional class III/IV, CCS class III/IV, or repetitive and disabling effort-related syncope. ASA was performed using previously described techniques [17]. Intracoronary echo-contrast agent was used for the evaluation of target septal branch for all patients undergoing ASA.

\section{Follow-up and definition of endpoints}

The primary endpoint was a composite of death from any cause and aborted cardiac arrest. The secondary endpoint was the invasive treatment of LVOTO during follow-up, including ASA, myectomy, and DDD pacing. The following conditions were considered to be cardiovascular death [18]: 1) procedural mortality of ASA, myectomy, 
and DDD pacing (deaths within 30 days after these procedures); 2 ) sudden cardiac death (SCD; unexpected within $1 \mathrm{~h}$ of witnessed collapse or nocturnal in previously stable patients); 3 ) heart failure-related death (in context of progressive cardiac decompensation); 4) stroke-related death (defined according to standard criteria [19]); or 5) fatal MI [20]. Death which occurred within 30 days after an invasive procedure is also counted as SCD, heart failure-related death, stroke-related death, or fatal MI, if corresponding criteria are met.

The follow-up of patients in the conservative group started at their first admission on January 1,2002 . For patients in the ASA group, the followup started on the day of operation. The endpoint status was ascertained by follow-up evaluation, including medical records, clinic visits, mailed questionnaires, telephone contact, short message, and Internet based instant messenger WeChat (Tencent, Inc., Shenzhen, Guangdong). For deceased patients, procurement of death certificates and interviews with next of kin was performed to determine the cause and time of death.

This study was approved by the local Ethics Committee with patient informed consent.

\section{Statistical analysis}

Continuous variables were expressed as mean \pm standard deviation (SD) or median (interquartile range $[\mathrm{IQR}]$ ), and differences between groups were analyzed by t test or Mann-Whitney U test, where appropriate. Categorical variables were summarized as frequencies with percentages and were compared by Pearson $\chi^{2}$ test, Fisher's exact test or continuity correction $\chi^{2}$ test, where appropriate. Univariate and multivariate Cox regression analyses were used to identify clinical predictors of endpoints. Variables with univariate $\mathrm{p}$ values $<0.10$ were selected for multivariate analysis and were expressed as hazard ratios (HRs) with 95\% confidence intervals (CIs). Multivariate Cox regression analysis was performed using backward stepwise method. The final number of variables in multivariate model was restricted according to the number of endpoint events to avoid overfitting. The following variables were evaluated: age, gender, coronary artery disease, hypertension, diabetes mellitus, atrial fibrillation, prior stroke, syncope/ presyncope, baseline left atrium diameter, baseline left ventricular diameter, baseline maximal septal thickness, baseline pressure gradient, and baseline ejection fraction. Kaplan-Meier survival curves were constructed to represent the survival graphically. Differences in survival between the groups were compared with log-rank test. When analyzing predictors of primary endpoint and comparing the survival free from primary endpoint, data on patients who received invasive treatment for LVOTO during follow-up period were censored at the date of their first procedure during the followup (i.e. the second invasive procedure for patients in ASA group, or the first invasive procedure for patients in conservative group). Data on patients who experienced aborted cardiac arrest were not censored at the date of resuscitation when analyzing predictors of secondary endpoint and comparing the survival free from secondary endpoint. All analyses were performed with SPSS statistical software, version 16.0 (SPSS Inc., Chicago, IL). All tests were 2 -tailed, and statistical significance was defined as a $\mathrm{p}<0.05$.

\section{Results}

\section{Baseline characteristics}

Medical records of 2,207 patients with $\mathrm{HCM}$ were screened. A total of 274 patients with HCM, severe drug-refractory symptoms, and a pressure gradient of $\geq 50 \mathrm{~mm} \mathrm{Hg}$ met the include/exclude criteria of this study and were all enrolled, 229 patients in the ASA group, 45 in the conservative group. Table 1 lists the baseline characteristics of patients in ASA group (age: $49.8 \pm 9.7$ years; $62.4 \%$ male) and conservative group (age: $52.5 \pm$ \pm 11.8 years; $60.0 \%$ male). The prevalence of NYHA class III/IV dyspnea, CCS class III/IV angina, and syncope/presyncope was similar between the two groups. No significant differences of baseline LVOT pressure gradient, medications, and comorbidities were observed.

\section{Acute procedural results and \\ 30-day periprocedural complications}

Septal ablation resulted in a median residual resting LVOT gradient of $25.0 \mathrm{~mm} \mathrm{Hg}$ (IQR $30.0 \mathrm{~mm} \mathrm{Hg})$. Two $(0.9 \%)$ patients in the ASA group died from procedural complications of ASA. One died from ventricular fibrillation (VF), the other for hemorrhagic shock caused by retroperitoneal hematoma. VF occurred immediately after alcohol injection in 2 patients, both of whom were successfully resuscitated. VF attacked another patient 2 days after ASA, who was also successfully resuscitated. No more cardiac arrests occurred in these 3 patients and they were still alive at the last follow-up. The leakage of alcohol into communicating branches resulted in acute $\mathrm{MI}$ beyond target region in $2(0.9 \%)$ patients; 1 died from VF 11 days after 
Table 1. Baseline variables.

\begin{tabular}{lccc}
\hline & Conservative & ASA & P \\
& $(\mathbf{n}=45)$ & $(\mathbf{n}=229)$ & 0.105 \\
\hline Age [years] & $52.5 \pm 11.8$ & $49.8 \pm 9.7$ & 0.757 \\
Male & $27(60.0 \%)$ & $143(62.4 \%)$ & 0.329 \\
NYHA class III/IV & $20(44.4 \%)$ & $120(52.4 \%)$ & 0.936 \\
CCS class III/IV & $16(35.6 \%)$ & $80(34.9 \%)$ & 0.138 \\
Syncope/presyncope & $16(35.6 \%)$ & $109(47.6 \%)$ & 0.093 \\
Atrial fibrillation & $8(17.8 \%)$ & $19(8.3 \%)$ & 0.857 \\
Prior stroke & $2(4.4 \%)$ & $6(2.6 \%)$ & 0.868 \\
Hypertension & $13(28.9 \%)$ & $69(30.1 \%)$ & 0.626 \\
Diabetes mellitus & $1(2.2 \%)$ & $12(5.2 \%)$ & $>0.999$ \\
Coronary artery disease & $4(8.9 \%)$ & $22(9.6 \%)$ & 0.076 \\
Maximum septal thickness [mm] & $21.1 \pm 3.4$ & $22.3 \pm 4.3$ & 0.024 \\
End-diastolic diameter [mm] & $43.7 \pm 5.7$ & $41.7 \pm 5.3$ & 0.001 \\
LVEF [mm] & $67.2 \pm 8.5$ & $71.6 \pm 7.8$ & 0.156 \\
Left atrium [mm] & $43.3 \pm 7.8$ & $41.5 \pm 6.2$ & 0.856 \\
Resting LVOT pressure gradient [mm Hg] & $90.4 \pm 35.5$ & $91.5 \pm 36.0$ & \\
Baseline medications: & & & 0.211 \\
$\quad$ Beta-blocker & $41(91.1 \%)$ & $192(83.8 \%)$ & 0.061 \\
Calcium channel blocker & $18(40.0 \%)$ & $60(26.2 \%)$ & \\
\hline ASA alcono & & \\
\hline
\end{tabular}

ASA - alcohol septal ablation; CCS — Canadian Cardiovascular Society; LVEF — left ventricular ejection fraction; LVOT — left ventricular outflow tract; NYHA — New York Heart Association; SD — standard deviation

the procedure as mentioned above, the other was still alive at the most recent follow-up. Complete atrioventricular block occurred in $101(44.1 \%)$ patients (Table 2). Nevertheless, atrioventricular conduction restored in all of these patients before discharge, and no new permanent pacemaker dependency occurred. Cardiac tamponade occurred in $2(0.9 \%)$ patients and they were all successfully treated with pericardiocentesis.

\section{Endpoints}

The follow-up was completed in $274(100 \%)$ patients with a median follow-up of 4.3 years (mean: 4.9 years; range: 3 days to 12.6 years; a total of 1,344 patient-years). As shown in Table 3, primary endpoint occurred in $21(7.7 \%)$ patients during the follow-up period (1.6\% per year), 8 in the conservative group (4.0\% per year), and 13 in the ASA group (1.1\% per year). The occurrence of in-hospital primary endpoint of the ASA group was described above. No in-hospital mortality happened in the conservative group. Two patients in the ASA group received implantable cardioverter defibrillator implantation during the follow-up, but no discharge events occurred. A total of
Table 2. Procedure data $(n=229)$.

\begin{tabular}{lc}
\hline Variables & Value \\
\hline $\begin{array}{l}\text { Residual resting pressure gradient, } \\
\text { median (IQR) [mm Hg] }\end{array}$ & $25.0(30.0)$ \\
Reduction in LVOT pressure & $71.6(19.0)$ \\
gradient (SD) [\%] & \\
Complete atrioventricular block & $101(44.1 \%)$ \\
Cardiac tamponade & $2(0.9 \%)$ \\
Ablated branches: & \\
First septal branch & $166(72.5 \%)$ \\
Second septal branch & $81(35.4 \%)$ \\
Third septal branch & $11(4.8 \%)$ \\
Fourth septal branch & $1(0.4 \%)$ \\
Second diagonal branch & $3(1.3 \%)$ \\
Branch of left circumflex & $2(0.9 \%)$ \\
Number of ablated branches: & \\
One & $196(85.6 \%)$ \\
Two & $31(13.5 \%)$ \\
Three & $2(0.9 \%)$ \\
Volume of alcohol injected, & $2.4(1.6)$ \\
median (IQR) [mL] & \\
\hline
\end{tabular}

IQR - interquartile range; LVOT — left ventricular outflow tract; SD - standard deviation 
Table 3. Endpoint.

\begin{tabular}{lccc}
\hline & Conservative & ASA & P \\
& $(\mathbf{n}=45)$ & (n = 229) & 0.013 \\
\hline Primary endpoint & $8(17.8 \%)$ & $13(5.7 \%)$ & 0.003 \\
All-cause mortality & $8(17.8 \%)$ & $10(4.4 \%)$ & 0.001 \\
Cardiovascular death & $8(17.8 \%)$ & $9(3.9 \%)$ & 0.417 \\
$\quad$ Sudden cardiac death & $1(2.2 \%)$ & $2(0.9 \%)$ & 0.001 \\
Heart failure-related death & $5(11.1 \%)$ & $2(0.9 \%)$ & 0.514 \\
Stroke-related death & $1(2.2 \%)$ & $3(1.3 \%)$ & $\mathrm{NA}$ \\
Alcohol septal ablation-related death & $\mathrm{NA}$ & $2(0.9 \%)$ & 0.164 \\
Fatal myocardial infarction & $1(2.2 \%)$ & $0(0 \%)$ & $>0.999$ \\
Non-cardiovascular death & $0(0 \%)$ & $1(0.4 \%)$ & $>0.999$ \\
Cancer-related death & $0(0 \%)$ & $1(0.4 \%)$ & $>0.999$ \\
Aborted cardiac arrest & $0(0 \%)$ & $3(1.3 \%)$ & 0.009 \\
Secondary endpoint & $9(20.0 \%)$ & $15(6.6 \%)$ & 0.034 \\
Alcohol septal ablation & $4(8.9 \%)$ & $4(1.7 \%)$ & $>0.999$ \\
Myectomy & $2(4.4 \%)$ & $10(4.4 \%)$ & $1(0.4 \%)$ \\
Dual chamber pacing & $3(6.7 \%)$ & 0.015 \\
\hline
\end{tabular}

Primary endpoint is a composite of death from any cause and aborted cardiac arrest. Secondary endpoint is the invasive treatments of left ventricular outflow obstruction during follow-up, including alcohol septal ablation, myectomy, and dual chamber pacing; ASA — alcohol septal ablation; NA - not applicable

Table 4. Predictors of primary and secondary endpoints.

\begin{tabular}{|c|c|c|c|c|}
\hline & \multicolumn{2}{|c|}{ Univariate } & \multicolumn{2}{|c|}{ Multivariate } \\
\hline & HR (95\% Cl) & $\mathbf{P}$ & HR (95\% Cl) & $\mathbf{P}$ \\
\hline \multicolumn{5}{|l|}{ Primary endpoint } \\
\hline Age & $1.01(0.97-1.06)$ & 0.632 & - & - \\
\hline Female & $2.07(0.84-5.10)$ & 0.115 & - & - \\
\hline Alcohol septal ablation & $0.31(0.12-0.78)$ & 0.013 & $0.22(0.08-0.60)$ & 0.003 \\
\hline Maximal septal thickness & $1.11(1.00 *-1.22)$ & 0.041 & $1.14(1.03-1.27)$ & 0.011 \\
\hline \multicolumn{5}{|l|}{ Secondary endpoint } \\
\hline Age & $1.00 *(0.96-1.04)$ & 0.938 & - & - \\
\hline Female & $0.58(0.23-1.47)$ & 0.254 & - & - \\
\hline Alcohol septal ablation & $0.28(0.12-0.64)$ & 0.003 & $0.25(0.11-0.59)$ & 0.001 \\
\hline Syncope/presyncope & $2.43(1.04-5.68)$ & 0.040 & $2.68(1.14-6.29)$ & 0.023 \\
\hline
\end{tabular}

Primary and secondary endpoints are defined as in Table 3; $\mathrm{Cl}$ - confidence interval; $\mathrm{HR}$ - hazard ratio; * $>1.00$

24 patients underwent invasive procedures to treat LVOTO during the follow-up, 15 in the ASA group, and 9 in the conservative group. No periprocedural mortality occurred after these procedures. One patient in the conservative group who received DDD pacing at 1.6 years of follow-up died at 7.4 years of follow-up. One patient in ASA group who received the second ASA procedure at 1.7 years of follow-up died at 8.9 years of follow-up. Other 22 patients who underwent invasive procedures to treat LVOTO during follow-up were still alive at the last follow-up.

Cox proportional analysis showed that the independent predictors of primary endpoint were ASA therapy (HR 0.22; 95\% CI 0.08-0.60; $\mathrm{p}=0.003$ ) and maximal septal thickness (HR 1.14; 95\% CI $1.03-1.27 ; \mathrm{p}=0.011$ ). Independent predictors of secondary endpoint were ASA therapy (HR $0.25 ; 95 \%$ CI $0.11-0.59 ; \mathrm{p}=0.001)$ and syncope/presyncope (HR 2.68; 95\% CI 1.14-6.29; $\mathrm{p}=0.023$ ) (Table 4). 


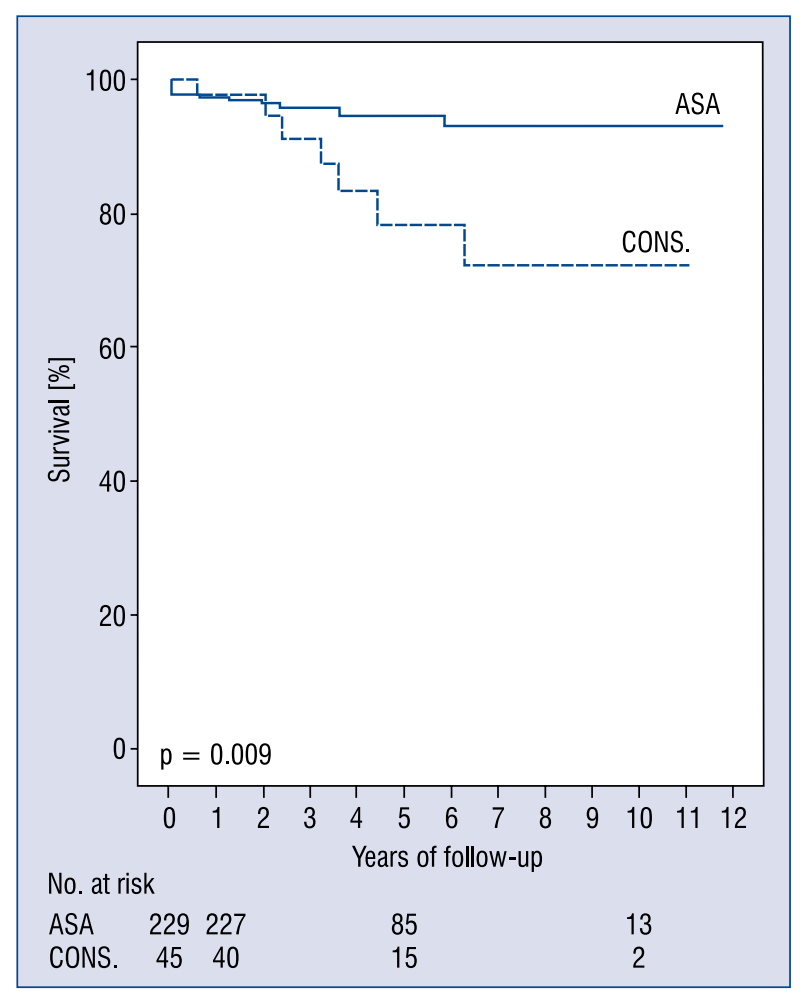

Figure 1. Survival free from primary endpoint after alcohol septal ablation (ASA) compared with the conservative (CONS.) group (log-rank $p=0.009$ ). Primary endpoint is a composite of death from any cause and aborted cardiac arrest.

Kaplan-Meier estimates of survival free from primary and secondary endpoints are shown in Figures 1 and 2 . The 1 -year survival free from primary endpoint of the ASA group was slightly numerically lower than that of the conservative group $(97.4 \%$ vs. $97.7 \%)$. The 5 - and 10 -year survival rates free from primary endpoint in the ASA $(94.5 \%$ and $93.0 \%$, respectively) group were greater than that in the conservative group (78.3\% 5-year, and $72.2 \%$ at 10 years; log-rank $\mathrm{p}=0.009$ ) (Fig. 1 ). The ASA group also experienced superior survival free from secondary endpoint than the conservative group (98.2\% 1-year, $93.8 \% 5$-year, and $88.7 \%$ at 10 years vs. $91.0 \% 1$-year, $77.7 \% 5$-year, and $77.7 \%$ at 10 years; log-rank $\mathrm{p}=0.001$ ) (Fig. 2 ).

\section{Discussion}

This report describes the survival after ASA compared with conservative therapy in patients with HCM, severe drug-refractory limiting symptoms, and a pressure gradient of $\geq 50 \mathrm{~mm} \mathrm{Hg}$ at rest or with provocation at a tertiary referral center

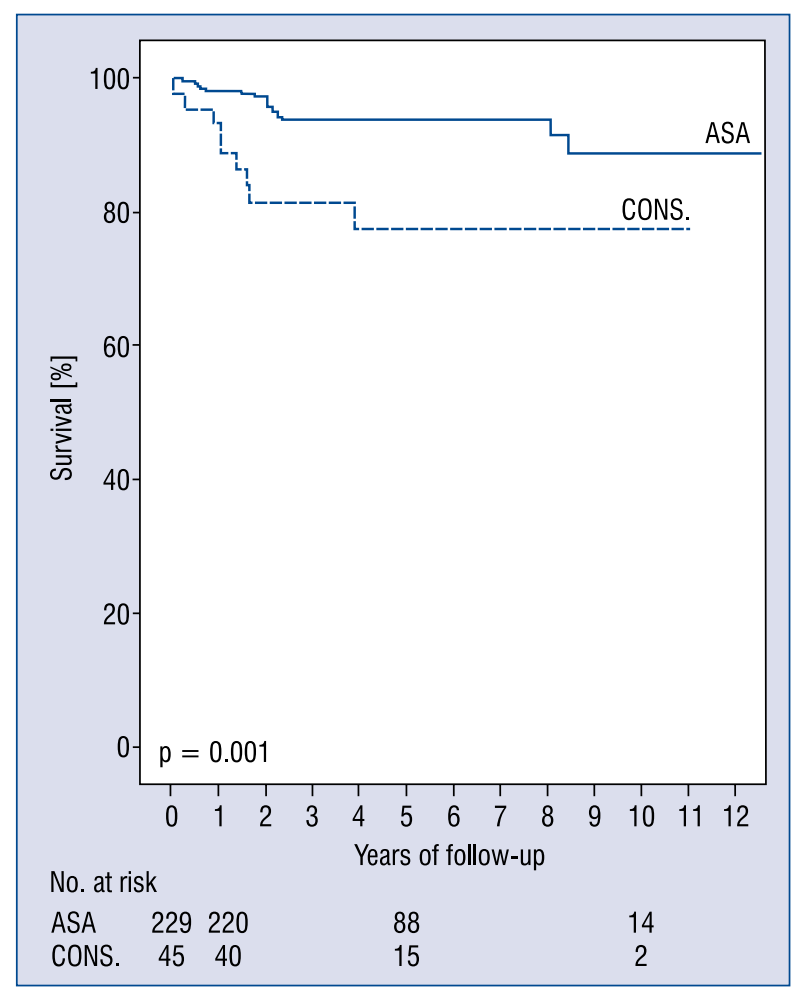

Figure 2. Survival free from secondary endpoint of the alcohol septal ablation (ASA) group compared with the conservative (CONS.) group (log-rank $p=0.001$ ). Secondary endpoint is the invasive treatments of left ventricular outflow obstruction during follow-up, including ASA, myectomy, and dual chamber pacing.

in China. The main findings of our study are: 1) survival of patients treated with ASA is better than that of conservative therapy; 2) ASA is independently associated with survival.

Alcohol septal ablation, an interventional technique designed to improve limiting symptoms by inducing relief of LVOTO, has been applied for nearly 20 years [16]. However, we are still not sure whether this technique extends longevity of its target patients. Results from several studies suggest that ASA can extend longevity of patients with HCM [6-12]. Here, we extended evidence for this notion by directly comparing the survival of patients undergoing ASA vs. a group of conservatively treated patients with comparable pressure gradient and limiting symptoms.

Periprocedural mortality of ASA in this study $(0.9 \%)$ was low and comparable to that of other tertiary referral centers $[8,11,21,22]$. No further lethal ventricular arrhythmia occurred in the three successfully resuscitated patients during follow-up suggested the prognostic significance of periprocedural cardiac arrest was limited, which agreed with 
the role of VF complicating acute MI [21, 23, 24]. Additionally, the numerically lower 1-year survival free from primary endpoint of ASA vs. conservative group ( $97.4 \%$ vs. $97.7 \%$ ) was associated with mortality and aborted cardiac arrest occurred during the perioperative period in ASA group, whereas, in the conservative group, no in-hospital primary endpoint occurred.

The relatively better survival of the ASA group in this study vs. several previous reports may be explained by the fact that patients in the ASA group of our study were younger (mean age: 49.8 years) than those from the other studies (mean age: 54-64 years) [8, 25-27]. There were reports demonstrating the survival advantage of patients younger than 60 years or 65 years [25, 28]. Besides, survival of the ASA group in this study was comparable to one cohort with a mean age of $<50$ years, which supports this viewpoint as well [29]. It is not surprising that the survival of the conservative group was not only worse than the one of the ASA group in this study, but also worse than conservatively treated groups in two recent studies [11, 12]. In both studies, the conservatively treated patients were less symptomatic and with lower pressure gradient. The survival advantage of ASA vs. conservative group suggests the survival disadvantage associated with LVOTO can be offset, at least partially, by ASA [3].

The incidence of new permanent pacemaker implantation in this study was low $(0 \%)$. This may be associated with the applying of myocardial contrast echocardiography and the prudent use of alcohol during ASA. Moreover, ASA is only performed by or performed under the guidance of the most experienced invasive operators in our institution, ensuring high safety of the procedure.

Several studies suggested age as an independent predictor of adverse events [8, 21, 25, 28], whereas Cox regression analysis did not identify this variable as an independent risk factor of primary or secondary end point in this study, which was in line with Veselka et al. [29]. We should note that age-related adverse events mainly occurred in patients older than 60 years [8, 25, 28]. Moreover, the mean age of our study population at enrollment was only about 50 years. This meant that the majority of our patients were still less than 60 years of age after a median follow-up of 4.3 years. Consequently, the role of age as an independent risk factor may not emerge in this age group.

No wonder a higher proportion of patients in the conservative group underwent invasive procedures to treat LVOTO during the follow-up.
These patients finally chose to receive invasive therapies because of disease progression, change in attitude, or getting enough money to afford invasive treatments. Interestingly, in the conservative group, the proportions of patients undergoing ASA, myectomy, or DDD pacing during the follow-up did not differ much, whereas, in the ASA group, there were obviously more patients who chose to receive myectomy than patients who chose to receive ASA and DDD pacing. This suggested a treatment preference shift after the first ASA procedure. Patients who experienced ASA seem more likely to choose myectomy when they need a second septal reduction procedure. This should be associated with the fact that myectomy has been demonstrated to bring a better relief of LVOTO $[4,5]$.

\section{Limitations of the study}

Some study limitations have to be acknowledged. First, this was a nonrandomized, retrospective cohort study. However, it is unlikely that a prospective randomized controlled trial comparing the outcome of conservative therapy vs. ASA will ever be conducted [30]. Second, as our data were retrieved from a tertiary referral center, there might also be a referral bias. The procedural results reported here represent those achieved by an experienced ASA referral center and therefore constitute a measure of the most favorable outcome that can be expected from ASA. It is inappropriate to extrapolate these findings to low-volume ASA centers. Third, the sample size of conservative group was relatively small. This is because only a very small proportion of patients who were indicated to invasive treatments will choose to give up or refuse these procedures in clinical practice. Finally, as disopyramide is not available in China, no patients in our study were treated with this medication.

\section{Conclusions}

This study suggests that in patients with HCM, severe drug-refractory symptoms and significant LVOTO, survival after ASA is favorable and better than that in patients treated conservatively. Thus, ASA seems to improve survival.

Funding sources: This work was supported by the National Science and Technology Major Project (No. 2012ZX09303008-001) of the Ministry of Science and Technology of China.

Conflict of interest: None declared 


\section{References}

1. Maron BJ, Gardin JM, Flack JM, Gidding SS, Kurosaki TT, Bild DE. Prevalence of hypertrophic cardiomyopathy in a general population of young adults. Echocardiographic analysis of 4111 subjects in the CARDIA Study. Coronary Artery Risk Development in (Young) Adults. Circulation, 1995; 92: 785-789.

2. Maron MS, Olivotto I, Zenovich AG et al. Hypertrophic cardiomyopathy is predominantly a disease of left ventricular outflow tract obstruction. Circulation, 2006; 114: 2232-2239.

3. Maron MS, Olivotto I, Betocchi S et al. Effect of left ventricular outflow tract obstruction on clinical outcome in hypertrophic cardiomyopathy. N Engl J Med, 2003; 348: 295-303.

4. Gersh BJ, Maron BJ, Bonow RO et al. 2011 ACCF/AHA Guideline for the Diagnosis and Treatment of Hypertrophic Cardiomyopathy: a report of the American College of Cardiology Foundation/American Heart Association Task Force on Practice Guidelines. Developed in collaboration with the American Association for Thoracic Surgery, American Society of Echocardiography, American Society of Nuclear Cardiology, Heart Failure Society of America, Heart Rhythm Society, Society for Cardiovascular Angiography and Interventions, and Society of Thoracic Surgeons. J Am Coll Cardiol, 2011; 58: e212-e260. doi: 10.1016/j. jacc.2011.06.011.

5. Elliott PM, Anastasakis A, Borger MA et al. 2014 ESC Guidelines on diagnosis and management of hypertrophic cardiomyopathy: The Task Force for the Diagnosis and Management of Hypertrophic Cardiomyopathy of the European Society of Cardiology (ESC). Eur Heart J, 2014; 35: 2733-2779. doi: 10.1093/ eurheartj/ehu284.

6. Sorajja P, Valeti U, Nishimura RA et al. Outcome of alcohol septal ablation for obstructive hypertrophic cardiomyopathy. Circulation, 2008; 118: 131-139. doi: 10.1161/CIRCULATIONAHA.107.738740.

7. Agarwal S, Tuzcu EM, Desai MY et al. Updated meta-analysis of septal alcohol ablation versus myectomy for hypertrophic cardiomyopathy. J Am Coll Cardiol, 2010; 55: 823-834. doi: 10.1016/j. jacc.2009.09.047.

8. Sorajja P, Ommen SR, Holmes DJ et al. Survival after alcohol septal ablation for obstructive hypertrophic cardiomyopathy. Circulation, 2012; 126: 2374-2380. doi: 10.1161/CIRCULATIONAHA.111.076257.

9. Jensen MK, Almaas VM, Jacobsson L et al. Long-term outcome of percutaneous transluminal septal myocardial ablation in hypertrophic obstructive cardiomyopathy: A Scandinavian multicenter study. Circ Cardiovasc Interv, 2011; 4: 256-265. doi: 10.1161/ CIRCINTERVENTIONS.110.959718.

10. Seiler C, Hess OM, Schoenbeck M et al. Long-term follow-up of medical versus surgical therapy for hypertrophic cardiomyopathy: A retrospective study. J Am Coll Cardiol, 1991; 17: 634-642.

11. Ball $\mathrm{W}$, Ivanov J, Rakowski $\mathrm{H}$ et al. Long-term survival in patients with resting obstructive hypertrophic cardiomyopathy comparison of conservative versus invasive treatment. J Am Coll Cardiol, 2011; 58: 2313-2321. doi: 10.1016/j.jacc.2011.08.040.

12. Vriesendorp PA, Liebregts M, Steggerda RC et al. Long-term outcomes after medical and invasive treatment in patients with hypertrophic cardiomyopathy. J Am Coll Cardiol Heart Fail, 2014; 2: 630-636. doi: 10.1016/j.jchf.2014.06.012.

13. Gersh BJ, Maron BJ, Bonow RO et al. 2011 ACCF/AHA Guideline for the Diagnosis and Treatment of Hypertrophic Cardiomyopathy: A report of the American College of Cardiology Foundation/American Heart Association Task Force on Practice Guidelines. Developed in collaboration with the American Association for Thoracic Surgery, American Society of Echocardiography, American Society of Nuclear Cardiology, Heart Failure
Society of America, Heart Rhythm Society, Society for Cardiovascular Angiography and Interventions, and Society of Thoracic Surgeons. J Am Coll Cardiol, 2011; 58: e212-e260. doi: 10.1016/j. jacc.2011.06.011.

14. Rakowski H, Sasson Z, Wigle ED. Echocardiographic and Doppler assessment of hypertrophic cardiomyopathy. J Am Soc Echocardiogr, 1988; 1: 31-47.

15. Sasson Z, Yock PG, Hatle LK, Alderman EL, Popp RL. Doppler echocardiographic determination of the pressure gradient in hypertrophic cardiomyopathy. J Am Coll Cardiol, 1988; 11: 752-756.

16. Sigwart U. Non-surgical myocardial reduction for hypertrophic obstructive cardiomyopathy. Lancet, 1995; 346: 211-214.

17. Yuan J, Qiao S, Zhang Y et al. Follow-up by cardiac magnetic resonance imaging in patients with hypertrophic cardiomyopathy who underwent percutaneous ventricular septal ablation. Am J Cardiol, 2010; 106: 1487-1491. doi: 10.1016/j.amjcard.2010.07.017.

18. Maron BJ, Olivotto I, Spirito P et al. Epidemiology of hypertrophic cardiomyopathy-related death: Revisited in a large non-referral-based patient population. Circulation, 2000; 102: 858-864.

19. The World Health Organization MONICA Project (monitoring trends and determinants in cardiovascular disease): A major international collaboration. WHO MONICA Project Principal Investigators. J Clin Epidemiol, 1988; 41: 105-114.

20. Pitt B, Pfeffer MA, Assmann SF et al. Spironolactone for heart failure with preserved ejection fraction. N Engl J Med, 2014; 370: 1383-1392. doi: 10.1056/NEJMoa1313731.

21. Veselka J, Krejci J, Tomasov P, Zemanek D. Long-term survival after alcohol septal ablation for hypertrophic obstructive cardiomyopathy: A comparison with general population. Eur Heart J, 2014; 35: 2040-2045. doi: 10.1093/eurheartj/eht495.

22. Jensen MK, Prinz C, Horstkotte D et al. Alcohol septal ablation in patients with hypertrophic obstructive cardiomyopathy: Low incidence of sudden cardiac death and reduced risk profile. Heart, 2013; 99: 1012-1017. doi: 10.1136/heartjnl-2012-303339.

23. Jensen GV, Torp-Pedersen C, Hildebrandt P et al. Does in-hospital ventricular fibrillation affect prognosis after myocardial infarction? Eur Heart J, 1997; 18: 919-924.

24. Bougouin W, Marijon E, Puymirat E et al. Incidence of sudden cardiac death after ventricular fibrillation complicating acute myocardial infarction: A 5-year cause-of-death analysis of the FAST-MI 2005 registry. Eur Heart J, 2014; 35: 116-122. doi: 10.1093/eurheartj/eht453.

25. Kwon DH, Kapadia SR, Tuzcu EM et al. Long-term outcomes in high-risk symptomatic patients with hypertrophic cardiomyopathy undergoing alcohol septal ablation. J Am Coll Cardiol Cardiovasc Interv, 2008; 1: 432-438. doi: 10.1016/j.jcin.2008.05.009.

26. Jensen MK, Havndrup O, Hassager C et al. Survival and sudden cardiac death after septal ablation for hypertrophic obstructive cardiomyopathy. Scand Cardiovasc J, 2011; 45: 153-160. doi: 10.3109/14017431.2011.565793.

27. Ten CF, Soliman OI, Michels M et al. Long-term outcome of alcohol septal ablation in patients with obstructive hypertrophic cardiomyopathy: A word of caution. Circ Heart Fail, 2010; 3: 362-369. doi: 10.1161/CIRCHEARTFAILURE.109.862359.

28. Kuhn H, Lawrenz T, Lieder F et al. Survival after transcoronary ablation of septal hypertrophy in hypertrophic obstructive cardiomyopathy (TASH): A 10 year experience. Clin Res Cardiol, 2008; 97: 234-243.

29. Veselka J, Krejci J, Tomasov $P$ et al. Survival of patients $\leq 50$ years of age after alcohol septal ablation for hypertrophic obstructive cardiomyopathy. Can J Cardiol, 2014; 30: 634-638. doi: 10.1016/j. cjca.2014.03.041.

30. Olivotto I, Ommen SR, Maron MS, Cecchi F, Maron BJ. Surgical myectomy versus alcohol septal ablation for obstructive hypertrophic cardiomyopathy. Will there ever be a randomized trial? J Am Coll Cardiol, 2007; 50: 831-834. 